\title{
Expert System for Magnetic Systems Investigations
}

\author{
S. Lima, R.V. Poliakova, F. Fernandez Nodarse, I.P. Yudin, \\ Joint Institute for Nuclear Research \\ 141980, Dubna, Muscow Region, Russia
}

\section{Abstract}

The paper deals with development of the conception and main functions of the system based on knowledge for magnetic systems investigations. The use of distributed artificial intelligence and cooperating systems is discussed. Some applications are presented.

\section{INTRODUCTION}

Distributed problem solving architectures can now also give benefits for intelligent advice giving and problem solving systems, as they might be used in accelerator operation and design. At point of view of the solution methods, this task was defined as a problem characterized by incomplete and inexact data, the use of heuristic knowledge for defining part of the values of the model parameters and its following solution. A Computer Aided Assistants (integrated expert systems) have been developed to solve this tasks using the architecture of cooperating distributive systems. Data are used with user feedback to provide the modeling and to change the solution. Multilevel problem solving scheme is used. The comparison of the different solutions for the tasks or subtasks carries out using a problem solving model with the use of qualitative model. It describes the system regimens.

\section{ACCELERATOR OPERATION AND CONTROL SYSTEMS}

Accelerator operation is a task demanding technical competence, experience, diagnostic skill and judgement. The essential ingredients in any kind of accelerator control system are: timing system, equipment interfaces, networks, computers, software. The operator has to handle large anounts of information coming from several different kinds of systems, coordinate and use a limited number of tools simultaneously, make a general judgment of the situation, and take reasonable decisions. Therefore, as soon as reliable computers were available and the accelerators complicated enough, a part of the operator's duties was offloaded onto computers. This formulation already implies that there are several layers of control objectives (hierarchical structure):

- reflexive controls ( simple servo and regulatory controls or simple sequential and interlocking digital devices). It is a rule to keep reflexive control away from the top layers and for this, modern microprocessors can be very good tools. Here the major portions of the real-time programs run.
- reactive controls (adaptive control, failure diagnostics, safety systems and multivariable controls). They are the realm of the distributed computerized control system today

- tactical controls. Using digital models of the analogous process, "accelerator" physicists try to optimize the controls and the machine. At this level it should be possible to try (in the model) different optical configurations of the accelerators and the corresponding changes in magnet settings.

- strategic controls. These would be the scheduling of the run times, selecting optimum energies to run at, and related topics.

The subdivision into decision layers as in a hierarchically ordered system is not followed in all existing accelerator control systems. A modern accelerator control system contains distributed intelligence for reflexive and, if possible, reactive control tasks. The hierarchy of control is realized in layers of hardware interconnected by networks. The lowest layers takes care of reflexive controls, the upmost layer of tactical or strategic controls. The interfaces to the process are in some way standardized to ease both commissioning and maintenance. Real-time operating software has to be switched coinciding with external signals. Many levels of timing precision can be distinguished. This observation leads unavoidably to the idea of local electronics at the device in question and a bus to transport information to some intelligent controller. Not all information flows to the control room through the computer networks. Analog signals, TV pictures and in particular interlock circuits go via other routes.

The analysis of the tasks of an operator show that there are different levels of involvement/ expertise necessary during the operation of the accelerator. Task- dependent knowledge is more useful in problem solving. These levels can described in the following way:

- Training and testing.

- Operation in normal conditions. The basic tasks for the operator are monitoring the performance and adjusting parameters in case of slight fluctuations to optimize the operation.

- High level breakdown, i.e. troubleshooting of high level faults like false parameter setting, an entirely broken element, drifts, etc. These faults should be seen as breakdowns that can be diagnosed on a conceptual level that needs no reasoning about the control system. 
- Low level breakdown, i.e. troubleshooting of low level elements/ modules of the control system like wrong data in equipment driver tables or faulty electronic interface modules whichs are only resolved by a controls specialist.

\section{CONCEPTION AND MAIN FUNCTIONS}

The use of artificial intelligence and workstations in the accelerator operation and design has become standard practice [1]. Most expert system (ES) tools used in this field comprise an inference engine, a knowledge acquisition system, a knowledge base, an explanation system and a user interface. $\Lambda$ pplied knowledgc usually takes the form of "assistant" programs (CAA - Computer Aided Assistant).

ES may be used to integrate a big program complex oriented to modeling the accelerator, for example in the design of the modification of the spectrometrical magnet SP-40 at the EXCHARM facility [2]. This problem solving system (CAA) assists the user during the following steps: to prepare the input data, to integrate and store the information required, to model the different solutions, to select the best solution, and to check the solution. During the modeling the heuristic knowledge may be play a big role. A prototype of this CAA has developed using blackboard architecture and network workstations.

The blackboard-system essentially consists of three components: blackboard data structure (BB), knowledge sources (KS) and control modules (CM). BB-systems emphasize the use of multiple cooperating subESs, or KSs. The purpose of BB (global solution database) is to hold computational and solution data needed and produced by the subsystems. The BB was segmented into distinct levels of abstraction. It also describes the problem solving strategies and programs. Each KS is a small knowledge based problem solver, and its internal processes have only local effects, rather than causing potential interactions with the rest of the system. KSs assist to the user in the selection of the problem solving stratcgies. The problem solving behavior of a BB-system is determined by the $\mathrm{KS}$ application strategy encoded in the control modules. Basically the $\mathrm{CM}$ determines the blackboard region to focus on and the particular KS to work on that region. Graphic tools are included

The following tasks were important for ES ariented to accelerator operation and control: on-line data processing (analog and digital data); finding heuristic methods of diagnosis from human expert (surface knowledge); modeling, and calculations (deep knowledge); local checks using oscilloscopes, measurement tool, etc. (off-line information); visual checks and ummeasured information; logbook, chart, and drawing checks; wave from diagnosis; and Case Base storage (indexing, learning).

The goal of the accelerator operation and control can be achieved if the identified tasks cooperate with each other. Well defined autonomous tasks like alarm monitoring and treatment, equipment access, optimization, management of archives, beam diagnosis, and control system diagnosis, can be regarded in the context of accelerator operation as loosely coupled cooperating agents. They can perform tasks independently of each other and can send requests to other agents to work on the common goal of accelerator operation. The cooperational features on top of these subtasks must include the capability of reasoning on the current state of the task, the current state of other tasks, and how the interaction between these tasks influences the control within any particular task.

The increasing complexity of accelerator control systems raises the need for distributed processing systems. A high degree of physical distribution and modularity and the need for continuous modifications and updates can easily make such evolving complex systems inconsistent. These kinds of problems are the subject of Distributed Artificial Intelligence (DAI). Distribution of the intelligence allows one to free the host from trivialities. Once freed from the reflexive control, it can support the operator effectively maximizes reliability and efficiency. Effective partitioning maximizes reliability and efficiency. A closed loop works properly only if all time constants are adcquately chosen and this applies also if a man is part of the loop. The user interface has to be user friendly. To be effective, the system has to perform three functions on the incoming data stream:

- a reliable and accurate collection, fast enough in real time, is mandatory;

- the vast amount of data, possibly created in this way, has to be reduced and concentrated; and

- each system user, operator and maintenance teclmician, has to have the possibility of accessing the bits, the equipment or physics parameter that they need in real time, without interruption or excessive time lag.

Software clearly depends on the architecture of the control system. The software includes data preparation and data acquisition modules (build up configuration, compute optics \& beam parameters, trim model output); databases (reference data set, target data set, current data set, accelerator description); hardware interface modules (initialize/ set/ increment hardware, read accelerator parameters, measure beam parameters); feedback modules (compute modifications); and learning mechanism (archive/ improve accelerator description, update when improved). The console programs at the accelerator may be generally classified in three main groups: study programs (modeling: accelerator physics - model beam dynamics); model programs (accelerator component description - compute machine/ beam parameters - accelerator parameters description); and realtime application programs (calculate/ set/ read/ adjust accelerator parameters, measured/ read parameters).

The CAA AOC for accelerator operation and control includes the following basic components: accelerator's CAA, operator's CAI (Computer Aided Instruction), operator's CAA (that assists the operator during the operation in 
normal conditions), diagnostic ESs (that assist the operator during the high and low level breakdown), DBMS, and help, utilities and interface programs.

The control system (CS) gives the possibility of accessing the different accelerator elements in a standard way.

Drift in parameters and optimization is checked by a subsystem of the operator's CAA, using the applications software for measurements and control actions. Test which change control values are passed through it.

The control system scans the equipment and produces an unfiltered alarm list. The alarm expert subsystem of the operator's CAA can be considered as an intelligent interface to the control systcm and its alarm system in particular and could be responsible for processing alarm data for the operator. Then it will reduce the feedback loop via the operator, by eliminating useless, misleading and unnecessary information

The operator's CAI is an ES oriented to training and to testing their technical competence and experience.

The ES for diagnosing hardware and software problems in the modules of the control system is mainly based on deep knowledge, and uses DBMS to instantiate the models used in a session. The path of the control parameter goes via several front end computers (network workstations), transmission systems and microcomputers, and was piloted by generic and specific software for the equipment control.

The ES for diagnosing problems concerning the accelerator and the beam includes a data acquisition module that acquires and judges information about beam parameters. It reduces the uncertainty in the acquisition.

The equipment diagnosis expert system verifies the hypotheses developed by the expert system for diagnosing problems.

The information about the layout and function of the accelerator and control system is stored in the database (DBMS). It also serves to fill information into local and on-line data storage. In operation it can be used for checking reality against dcsign intentions, mostly for software modules, and for storage of reference data for well defined operational situations. The distributed database contains the translation table, the current values, set points and actual values for each piece of equipment, conversion factors, end point settings and many other parameters.

The user interface module coordinates information from the different subsystems and takes care of the presentation to the user. The operator interface includes the use of interactive menus, spreadsheets and distributed windows.

A framework [3] for integration of cooperating distributed systems in process supervision, design (modeling) and control applications in the accelerator was developed. The Cooperation Layer, that provides the means for cooperation, is decomposed into several components. The Intelligent. System (IS) incorporates subtasks of the problem. The Monitor is the control instance and connects the ISs or semi-autonomous processing elements to the Cooperation Layer. Using the knowledge sources, the Planning and Coordination Module supervises all cooperational behavior like situation assessment and planning. The Communication Modules and the Session Layer do all message passing between systems. The Information Management Module (DBMS) provides a distributed information access mechanism to support the remote access and sharing of information among systems and it is in close connection with the Acquaintance Models (AM) and the Self Model (SM). The SM Model represents a system's own capabilities, needs and interests. The AM stores similar information about other systems of the cooperating community in which the system itself is interested. The accelerator's CAA provides (integrates) the functions of the Cooperation Layer and Session Layer. This framework may also be used for each intelligent system.

These CAAs integrate systems based on knowledge, databases, spreadsheets, graphic tools and other utilities. It also provides a user-friendly interface. This conception was developed using network workstations for the MT-25 microtron [4] at JINR to help microton operator in the preparation, start, technical fault search and adjust, run (operation), turn off and maintenance processes .

\section{CONCLUSION}

Computer Aided Assistants based on knowledge and characterized by good ergonomic conditions, user-friendly interfaces and adequate response time make more easy the work, reduce the training time and the number of user mistakes. They use symbolic manipulation and numerical methods, heuristic knowledge and specialized graphic tools.

The proposed architecture of cooperating distributed systems increases the performance and power of such systems specially of the cooperating heterogeneous on-line systems for operating an accelerator. It covers the whole range of diagnosis, process optimization, recovery actions and unified presentation to the operator. This has emerged from the careful study of the accelerator operation at the JINR.

\section{REFERENCES}

[1] M. Lee, S. Clearwater, "GOLD: Integration of Modelbased Control Systems with Artificial Intelligence and Workstations," (SLAC-PUB-4396, August 1987).

[2] E.P. Zhidkov et al., "Mathematical Modeling of Some Modification of Spectrometrical Magnet SP-40," JINR Communication P11-92-490, Dubna, 1992.

[3] ARCHON - Architecture for Coopcrating Heteroge neous On-line Systems Status Report (compiled by S. Becker, J.Ehlers), ESPRIT Conference Week 1990, and Technical Report No.9 (ESPRIT - Project 2256 ARCHON) 9-1990.

[4] V.G. Ivanov et al., "Automated Workstation for Microtron MT-25. Conception and main functions," JINR Communication P10-91-302, Dubna, 1991. 